American Journal of Biochemistry and Biotechnology 2 (1): 19-24, 2006

ISSN 1553-3468

(C) 2006 Science Publications

\title{
Cardiovascular Risk Factors in North Indians: A Case-Control Study
}

\author{
${ }^{1}$ Vivek P. Singh, ${ }^{2}$ V. Ramesh, ${ }^{1}$ Sonal Somvanshi, ${ }^{3}$ Nakul Sinha, \\ ${ }^{3}$ Satyendra Tewari, and ${ }^{1}$ Suraksha Agrawal \\ ${ }^{1}$ Department of Medical Genetics, ${ }^{2}$ Department of Pathology, ${ }^{3}$ Department of Cardiology, Sanjay Gandhi \\ Post Graduate Institute of Medical Sciences, Raebareli Road, Lucknow (UP) 226014 India
}

\begin{abstract}
There is no large study from North India which has addressed association of (CAD) in angiographically proven cases with lipid levels and the conventional risk factors. In the present study risk factor assessment was done in 200 consecutive patients admitted for coronary angiography and in 200 age and sex matched controls. We have found that the most important predictor of CAD is current smoking (odds ratio [OR] 3.05, p $<0.0001$ ). In subjects with cases, the levels of mean lipid and apolipoprotein levels were significantly higher than controls. The younger CAD group, age ( $\leq 45$ years) had significantly higher levels of total cholesterol $(\mathrm{p}<0.001)$, LDL-cholesterol $(\mathrm{p}<0.001)$ and apolipoprotein B $(\mathrm{p}<0.04)$ than the older patients group, age $(>45$ years $)$, Diabetic CAD patients had significantly higher levels of total cholesterol $(\mathrm{p}=0.03)$ and triglycerides $(\mathrm{p}=0.005)$ than non-diabetics. The conventional risk factor in diabetics was hypertension and, in younger age group ( $\leq 45$ years), it was smoking and a family history of CAD. In univariate analysis, higher socioeconomic status (OR $0.30, \mathrm{p}=0.005$ highest vs. lowest; OR 0.73 middle vs. lowest) was observed. In the multiple logistic regression analysis only total cholesterol $(\mathrm{p}<0.01)$, HDL- cholesterol $(\mathrm{p}<0.01)$, apolipoprotein $\mathrm{B}$ $(\mathrm{p}<0.01)$, and smoking $(\mathrm{p}<0.01)$ had a significant independent association with CAD.

Smoking cessation, treatment of hypertension, the ATP-III guidelines for the treatment of dyslipidemia and improving the level of education are likely to have a profound effect on the burden of premature coronary artery disease in North India.
\end{abstract}

Key words: Coronary artery disease, risk factors, north-Indians

\section{INTRODUCTION}

A dramatic increase in the prevalence of CAD is predicted in the next 20 years, due to rapid change in demography and lifestyle ${ }^{[1]}$. In north India it has been reported that prevalence of coronary artery disease has increased in Urban India to ninefold over a period of 35 years $^{[2]}$, and in rural areas it has almost doubled in the last decade ${ }^{[3]}$ the exact cause is not known ${ }^{[4]}$. The prevalence of conventional risk factors such as smoking, hypertension and diabetes mellitus is not different in South Asians as compared with other ethnic groups ${ }^{[5]}$. Lipid patterns however, are known to vary with dietary habits, which could be different in diverse ethnic groups and geographical locations. Thus South Asians are noted to have higher triglyceride (TG) levels, low concentration of high-density lipoprotein cholesterol (HDL-c), increased visceral fat and higher insulin resistance as more prevalent risk factors ${ }^{[6]}$. While total cholesterol (TC) levels have been observed to be lower in CAD patients of Indian origin when compared to their counterparts in the west ${ }^{[6]}$. Most of these studies are based on migrant Indians and a few reports are from South India ${ }^{[7]}$. There is insufficient data on lipid levels in patients with known CAD from Indians living in India, and especially North India. We conducted a hospital-based-case-control study on patients with proven CAD from Uttar Pradesh, India to assess the relative importance of serum lipid patterns and other conventional risk factors for CAD among North Indians.

\section{METHODS}

Subjects: 200 consecutive patients admitted for coronary angiography at our hospital for typical or atypical chest pain or post-myocardial infarction assessment. Diagnosis of CAD was considered that all patients with electrocardiographic or echocardiographic evidence of myocardial infarction and those with obstructive lesions $(\geq 50 \%)$ on coronary angiography in any of the coronary arteries or their branches. All cases were enrolled between January 2004 and March 2005.

200 age and sex matched controls were evaluated during the same period, with their clinical history, physical examination. The negative treadmill test was

Corresponding Author: Prof. Suraksha Agrawal, Department of Medical Genetics, Sanjay Gandhi Post Graduate Institute of Medical Sciences, Lucknow, 226014, India, Tel: 091-522 -2668007, Ext 2338; Fax : 091-522 $2668973 / 2668017$ 
used as exclusion criteria for the presence of coronary artery disease.

Variables: We recorded data on socio demographic characteristics such as age, sex, marital status, occupation, monthly income, and educational level in all participants. Patients already taking antihypertensive medication or those whom the average of two blood pressure readings at least five minutes apart in the sitting posture was $>140 / 90 \mathrm{~mm}$ $\mathrm{Hg}$ were labelled as hypertensive. Diabetes mellitus was diagnosed to be present if a patient had a fasting glucose $\geq 126 \mathrm{mg} / \mathrm{dl}$ or two-hour post-load glucose $\geq$ $200 \mathrm{mg} / \mathrm{dl}$. Smoking habits were classified into who have never smoked, individuals who smoked earlier but left smoking within past one year and individuals who are currently smoking ( $>10$ cigarettes per day for at least 10 years). Individuals who admitted ingesting alcohol i.e. light-moderate alcohol intake (one to two drinks per day or $<30 \mathrm{~g} /$ day) were categorized as alcohol consuming. Anthropometrics measurements included height and weight measurements, and the body mass index (BMI) was calculated using the formula weight $(\mathrm{Kg}) /$ height $\left(\mathrm{m}^{2}\right)$. A family history of CAD was defined as myocardial infarction or sudden death before the age of 55 years in father or any other male first-degree relative, or before the age of 65 years in mother or any other female first-degree relative.

Lipids analysis: Fresh fasting samples (after 12 hours of overnight fasting) were used for the estimation of lipid profile. Patients receiving lipidlowering agents or having renal failure, hepatic or thyroid disorders or those presenting within 8 weeks of acute myocardial infarction, and patients taking noncardiac drugs which affect the lipid profile (such as steroids) were excluded from the study. Total cholesterol (TC) and triglycerides (TG) measured by the enzymatic method (Chemistry autoanalyzer, Technicon RX-XT make model). High-density lipoprotein cholesterol (HDL-C) concentrations were measured by enzymatic assay after phosphotungstic acid and magnesium precipitation. Commercially available reagents were used to measure TC, TG and HDL-C from Boehringer Mannheim (Germany). VLDL-cholesterol was estimated by dividing the TG levels by a factor of 5. LDL-cholesterol was obtained by subtracting the sum of VLDL-c and HDL-c fractions from TC by applying the Friedewald formula. Apolipoprotein B levels were assessed by the Immunoturbidimetric Immunoassay using commercial kit (Randox Laboratories Ltd. UK).

Subgroups: Patients with proven CAD were further divided into two subcategories. The first subgroup analysis was based on the age of the patients, i.e. age $\leq 45$ years vs. $>45$ years. The second subgroup analysis was based on the presence or absence of diabetes mellitus.

Statistical analysis: Statistical analysis was done using SPSS statistical software (10.0 versions). Discrete data was compared using Chi-square test and continuous data using Student's t test. Test of proportion was done to compare the risk factors between the two groups, i.e. diabetic vs. non-diabetic and age $\leq 45$ years vs. $\geq 45$ years. Differences among age, body mass index, lipid and lipoprotein concentrations in different groups of individuals were compared using the Student's t test. The distribution of triglyceride concentrations was skewed to the right; hence statistical tests were carried out on the logarithmic transformation of triglyceride levels. Multiple logistic regression analysis was performed using $\mathrm{CAD}$ as the dependent variable and the risk factors, which had a significant association with $\mathrm{CAD}$ in the univariate analysis, namely body mass index (BMI), systolic blood pressure, diastolic blood pressure total cholesterol, triglyceride, HDL-C, LDLC, VLDL-C, apolipoprotein B, smoking and alcohol, as the independent variable. $\mathrm{P}$ value of $\mathrm{P}<0.05$ was considered statistically significant.

\section{RESULTS}

Table 1 shows the Comparisons of the anthropometric, biochemical and socioeconomic characteristics of the two groups.

Table 1: Anthropometric, Biochemical and Socioeconomic Characteristics of CAD patients and controls.

\begin{tabular}{|c|c|c|c|}
\hline & $\begin{array}{l}\text { Cases } \\
(n=200)\end{array}$ & $\begin{array}{l}\text { Control } \\
(n=200)\end{array}$ & p value \\
\hline Age & $47.3 \pm 12.1$ & $44.5 \pm 13.3$ & --- \\
\hline $\operatorname{Sex}(M / F)$ & $169 / 31$ & $163 / 37$ & --- \\
\hline $\operatorname{BMI}\left(\mathrm{Kg} / \mathrm{m}^{2}\right)$ & $23.4 \pm 3.5$ & $21.8 \pm 4.1$ & 0.001 \\
\hline Waist: hip ratio & $0.94 \pm 0.05$ & $0.93 \pm 0.06$ & 0.03 \\
\hline $\begin{array}{l}\text { Systolic BP } \\
(\mathrm{mm} \mathrm{Hg})\end{array}$ & $131.2 \pm 15$ & $125.5 \pm 18$ & 0.026 \\
\hline $\begin{array}{l}\text { Diastolic BP } \\
(\mathrm{mm} \mathrm{Hg})\end{array}$ & $81.5 \pm 15$ & $77.0 \pm 12$ & 0.020 \\
\hline \multicolumn{4}{|l|}{ Lipids (mg/dl) } \\
\hline $\mathrm{TC}$ & $193 \pm 85$ & $142 \pm 65$ & $<0.0001$ \\
\hline TG & $177 \pm 48$ & $144 \pm 31$ & $<0.0001$ \\
\hline HDL-C & $30 \pm 9.0$ & $35 \pm 9.4$ & $<0.0001$ \\
\hline LDL-C & $105 \pm 45$ & $80 \pm 24$ & $<0.0001$ \\
\hline VLDL-C & $38 \pm 17$ & $35 \pm 12$ & NS \\
\hline TC / HDL ratio & $6.43 \pm 1.4$ & $4.06 \pm 1.3$ & 0.001 \\
\hline LDL / HDL ratio & $3.5 \pm 1.0$ & $2.29 \pm 1.1$ & 0.0022 \\
\hline Apo B (mg/dl) & $149 \pm 44$ & $103 \pm 41$ & $<0.0001$ \\
\hline \multicolumn{4}{|l|}{ Income/ month } \\
\hline$<$ Rest 5000 & $091(46.0)$ & $074(37.0)$ & 0.005 \\
\hline Rest $5000-10,000$ & $081(40.5)$ & $078(39.0)$ & \\
\hline Rest $\geq 10,000$ & $028(13.5)$ & $048(24.0)$ & \\
\hline $\begin{array}{l}\text { Educational } \\
\text { status }\end{array}$ & \multicolumn{2}{|c|}{ Educational } & 0.02 \\
\hline None & $119(59.5)$ & $099(49.5)$ & \\
\hline School & 055 (27.5) & 077 (38.5) & \\
\hline \multicolumn{4}{|l|}{ College } \\
\hline Married (\%) & $170(85.0)$ & $165(83.0)$ & NS \\
\hline
\end{tabular}


Among 200 cases, 68 (34\%) patients revealed the family history of CAD, 131 had angina, and 69 had a history of myocardial infarction. All these 200 subjects had CAD based on angiography, diabetes was present in $32(16 \%)$ of the patients out of which $13(6.5 \%)$ patient were having type I and $19(9.5 \%)$ patients were having type II diabetes. We have also analyzed hypertension in terms of systolic and diastolic blood pressure; out of 200 patients only 106 (53\%) had hypertension

Body mass index, waist-to-hip ratio and systolic and diastolic blood pressure were significantly higher in patients as compared to controls. All the lipid parameters, viz. TC, HDL-c, LDL-c, and TG were significantly higher in patients but there was no significant difference in the VLDL cholesterol. However, TC, TG and LDL-c lipid fraction levels were within the normal range and HDL-c levels which were lower than normal in both the groups. Nuptility was also similar in both the groups. Cases had significantly lower monthly incomes than controls $\left(\chi^{2}=10.64, \mathrm{p}=0.004\right)$, and very few under went college education $\left(\chi^{2}=4.98, \mathrm{p}=0.02\right)$. The Odds Ratio (OR) for CAD was 0.30 (95\% CI 0.14-0.64) for the highest versus lowest income group and 0.73 (0.45-1.15) for the middle versus lowest income groups.

Characteristics features of different life styles of patients and controls are shown in Table 2. Smoking was an important risk factor. 140 (70\%) cases and 90 (45\%) controls were current smokers (OR 2.8, 95\% CI 1.89-4.30).

Table 2: Lifestyle and diet of the study population

\begin{tabular}{llll}
\hline & $\begin{array}{c}\text { Case } \\
(\mathbf{n = 2 0 0})\end{array}$ & $\begin{array}{c}\text { Control } \\
(\mathbf{n}=\mathbf{2 0 0})\end{array}$ & P* \\
\hline Smokers* & & & \\
Never & $60(30 \%)$ & $110(55 \%)$ & $<0.0001$ \\
Former & $21(10 \%)$ & $25(12.5 \%)$ & 0.3191 \\
All current & $119(59.5 \%)$ & $65(32.5 \%)$ & $<0.0001$ \\
Current<10/day & $40(20 \%)$ & $42(21 \%)$ & 0.4500 \\
Current $\geq 10 /$ day & $79(39.5 \%)$ & $23(11.5 \%)$ & $<0.0001$ \\
Alcoholic & $89(44.5 \%)$ & $60(30 \%)$ & 0.003 \\
$(<30 \mathrm{~g} /$ day $)$ & & & \\
Diet & & $105(52.5 \%)$ & 0.066 \\
Veg & $89(44.5 \%)$ & $95(47.5 \%)$ & 0.066 \\
\hline
\end{tabular}

Never smoked at any time (never), smoked in the past (former), and any current smoker (current), values are n (\%)

It was seen that current smokers were higher in patients $119(59.5 \%)$ as compared to controls 65 $(32.5 \%)$ (P value $=0.0001$, OR 3.05, 95\% CI 2.024.59). There were a higher proportion of heavy smokers ( $\geq 10$ cigarettes/day) among cases than controls (39.5 vs. $11.5 \%$, OR 5.02, p < 0.0001, 95\% CI 2.99-8.44). Current smokers with CAD were having significantly higher total cholesterol $(\mathrm{p}=0.0001)$ and triglycerides $(\mathrm{p}=0.0001)$ while highdensity lipoprotein was lower $(\mathrm{p}<0.0001)$ in CAD. Although alcohol consumption was more in CAD $(89,45 \%)$ as compared to controls $(60,30 \%$, $\mathrm{p}=0.003$ ), when the data were adjusted for the effect of smoking, alcohol was not found to be a risk factor $(p=0.213)$. The high risk of CAD in Indians has been attributed to ghee (clarified butter) consumption, but in our study regular use of ghee was similar in the two groups $(18 / 140,13 \%$ in cases, $23 / 164,14 \%$ in controls; $p=0.712$ ). Individuals were classified into vegetarian or non-vegetarian by a simple questionnaire, non-vegetarian were higher in patients $111(55.5 \%)$ as compared to controls 95 (47.5\%), however, the differences were not significant (OR 0.72, $\mathrm{p}=0.06$ ).

Table 3 shows the stratification of proven CAD patients into young and more than 45 years of age, lipid profiles.

Table 3: Lipid profile and other conventional risk factors of patients with CAD of age $\leq 45$ years vs. age $>45$ years (test of proportions)

\begin{tabular}{lccl}
\hline $\begin{array}{l}\text { Lipid } \\
(\mathbf{m g} / \mathbf{d l})\end{array}$ & $\begin{array}{l}\text { Age } \leq \text { 45years } \\
\text { n=96 (mg/dl, } \\
\text { mean } \pm \text { SD) }\end{array}$ & $\begin{array}{c}\text { Age }>\text { 45,years, } \\
\text { n=104 (mg/dl, } \\
\text { mean } \pm \text { SD) }\end{array}$ & p value \\
\hline TC & $220 \pm 85$ & $181 \pm 80$ & $<0.001$ \\
TG & $190 \pm 48$ & $180 \pm 35$ & NS \\
HDL-C & $32 \pm 9.0$ & $30 \pm 9.4$ & NS \\
LDL-C & $122 \pm 41$ & $104 \pm 38$ & $<0.001$ \\
VLDL-C & $38 \pm 15$ & $36 \pm 12$ & NS \\
Apo-B & $151 \pm 42$ & $139 \pm 40$ & $<0.04$ \\
Risk factors & & & \\
\hline Hypertension (\%) & $40(41 \%)$ & $66(63 \%)$ & $0.003^{*}$ \\
Diabetes (\%) & $7(7 \%)$ & $25(24 \%)$ & $0.002^{*}$ \\
Family history & $43(45 \%)$ & $25(24 \%)$ & $0.003^{* *}$ \\
of CAD (\%) & & & \\
Smoking (\%) & $72(75 \%)$ & $47(45 \%)$ & $<0.0001^{* *}$ \\
Alcohol (\%) & $40(42 \%)$ & $49(47 \%)$ & NS \\
\hline
\end{tabular}

* Significant in favor of age $\leq 45$ years, ** significant in favor of age $>45$ years, $\mathrm{NS}=$ not significant.

Young CAD patients ( $\leq 45$ years) had significantly higher levels of total cholesterol $(\mathrm{p}<0.001)$, LDLcholesterol $(\mathrm{p}<0.001)$ and apolipoprotein B $(\mathrm{p}<0.04)$ than the older patients ( $>45$ years). However, there were no differences in TG levels, VLDL levels and HDL-cholesterol levels between the two groups.

Test of proportion was performed to see the effect of conventional risk factors on younger $(\leq 45)$ and $(>45)$ years CAD patients. Test of proportion revealed that a family history of CAD $(45 \%$ v. $24 \%$, $\mathrm{p}=0.003)$ and smoking $(75 \%$ v. $45 \%, \mathrm{p}<0.0001)$ were significantly higher in younger $(\leq 45$ years) than older 
( $>45$ years) patients but the prevalence of hypertension $(41 \%$ v. $63 \%, \mathrm{p}=0.003)$ and diabetes mellitus ( $7 \%$ v. 24\%, p=0.002) were significantly higher in older patients as compared to controls. CAD patients were stratified into diabetic and nondiabetics to see the lipid profile and role of other conventional risk factors (Table 4).

Table 4: Lipid profile and other Conventional risk factors of diabetics vs. non- diabetics in patients with CAD (Test of proportions)

\begin{tabular}{lccl}
$\begin{array}{l}\text { Lipid } \\
(\mathbf{m g} / \mathbf{d l})\end{array}$ & $\begin{array}{l}\text { Diabetics } \\
(\mathbf{n = 3 2}), \\
(\mathbf{m g} / \mathbf{d l}, \\
\text { mean } \pm \text { SD) }\end{array}$ & $\begin{array}{l}\text { Non- diabetics } \\
(\mathbf{n = 1 6 8}),\end{array}$ & $\begin{array}{l}\text { P } \\
\text { (mg/dl, } \\
\text { mean } \pm \text { SD })\end{array}$ \\
\hline TC & $214 \pm 82$ & $180 \pm 76$ & 0.030 \\
TG & $197 \pm 38$ & $175 \pm 42$ & 0.005 \\
HDL-C & $31 \pm 9.0$ & $30 \pm 9.4$ & NS \\
LDL-C & $109 \pm 41$ & $103 \pm 38$ & NS \\
VLDL-C & $39 \pm 15$ & $36 \pm 12$ & NS \\
Apo-B & $136 \pm 37$ & $134 \pm 40$ & NS \\
Risk factors & & & \\
\hline Smoking $(\%)$ & $15(46 \%)$ & $104(61 \%)$ & NS \\
F/H of CAD $(\%)$ & $12(37 \%)$ & $56(33 \%)$ & NS \\
Hypertension & $25(78 \%)$ & $81(48 \%)$ & 0.003 \\
\hline
\end{tabular}

$\mathrm{NS}=$ not significant

Diabetic patient had significantly higher level of total cholesterol $(\mathrm{p}=0.03)$ and triglycerides $(p=0.005)$ than non-diabetics. It is evident from the table that hypertension was seen in $78 \%$ of diabetic patients (CAD) as compared to non-diabetic CAD patients $(48 \%)$. While other risk factors like smoking and family history was not significantly different in diabetic vs. non-diabetic CAD patients.

Table 5: Multivariate logistic regression of determinants of CAD

\begin{tabular}{llllll}
\hline Variables & $\boldsymbol{\beta}$ & S.E. & $\begin{array}{l}\text { P } \\
\text { Value }\end{array}$ & OR $(\mathbf{9 5 \%}$ CI) \\
& & & & & \\
\hline BMI & 0.030 & 0.04 & 0.43 & 1.03 & $(1.0-1.1)$ \\
SBP & -0.0017 & 0.014 & 0.91 & 1.01 & $(0.97-1.03)$ \\
DBP & 0.0126 & 0.022 & 0.57 & 1.01 & $(0.9-1.06)$ \\
TC & $\mathbf{0 . 0 2 3 2}$ & $\mathbf{0 . 0 0 5}$ & $<\mathbf{0 . 0 1}$ & $\mathbf{1 . 0 2}$ & $(\mathbf{1 . 0 1 - 1 . 0 3})$ \\
TG & 0.004 & 0.003 & 0.17 & 1.004 & $(1.0-1.09)$ \\
HDL-C & $\mathbf{- 0 . 0 5 7}$ & $\mathbf{0 . 0 1 5 5}$ & $<\mathbf{0 . 0 1}$ & $\mathbf{1 . 0 6}$ & $(\mathbf{0 . 9 1 - 1 . 0 8})$ \\
LDL-C & 0.0090 & 0.0056 & 0.062 & 1.008 & $(1.0-1.02)$ \\
VLDL-C & 0.014 & 0.020 & 0.49 & 1.0 & $(0.95-1.03)$ \\
Apo- B & $\mathbf{0 . 0 1 5 4}$ & $\mathbf{0 . 0 0 3 3}$ & $<\mathbf{0 . 0 1}$ & $\mathbf{1 . 0 1}$ & $(\mathbf{1 . 0 0 - 1 . 0 2})$ \\
Smoking & $\mathbf{1 . 3 1 0 0}$ & $\mathbf{0 . 2 9 8}$ & $<\mathbf{0 . 0 1}$ & $\mathbf{3 . 7 2}$ & $(\mathbf{2 . 0 8}-6.70)$ \\
Alcohol & 0.034 & 0.042 & 0.44 & 1.02 & $(1.0-1.1)$ \\
\hline
\end{tabular}

Dependent variable: coronary artery disease; independent variables; body mass index (BMI), systolic blood pressure(SBP), diastolic blood pressure(DBP), total cholesterol (TC), triglyceride (TG), HDL-cholesterol (HDL), LDL-cholesterol (LDL), VLDLcholesterol (VLDL), apolipoprotein B (Apo B), smoking and alcohol. $\mathrm{CAD}=$ coronary artery disease; $\mathrm{CI}=$ confidence interval; $\beta=\beta$ coefficient S.E. $=$ standard error, $\mathrm{OR}=$ odds ratio.
Multiple logistic regression analysis was performed using CAD as the dependent variable and the risk factors, which had a significant association with $\mathrm{CAD}$ in the univariate analysis, namely body mass index (BMI), systolic blood pressure, diastolic blood pressure, TC, triglyceride, HDL-C, LDL-C, VLDL-C, apolipoprotein B, smoking and alcohol, as the independent variables as shown in (Table 5). Only TC (odds ratio [OR]: 1.02, p<0.01), HDL- C (OR: 1.06, p<0.01), apolipoprotein B (OR: 1.01, $\mathrm{p}<0.01$ ), and smoking (OR: 3.72, $\mathrm{p}<0.01)$ had a significant association with CAD.

\section{DISCUSSION}

India is in a phase of transition in which infectious and nutritional diseases are the major causes of morbidity and mortality. One disease of major impact is $\mathrm{CAD}^{[8]}$. The large population of the country, the problem of CAD will be a major source of death and disability, as well as a major drain on national resources. The reasons for this rise in the incidence of CAD among urban Indians are the subjects of discussion but also require more research.

Our study, which is probably the first prospective case-control study aimed to identify the relation between risk factors and coronary artery disease in North Indians. Since the cases are confirmed cases of CAD not known to have had previous heart disease, the study avoids the problem of misdiagnosis associated with sole use of ECG as a diagnostic criteria (as has been done in prevalence studies) and of modification of risk factors by treatment of CAD.

Cigarette smoking is important modifiable risk factors for CAD in western countries ${ }^{[9]}$. Studies on migrant Indian populations have not emphasized the importance of smoking ${ }^{[6]}$. Our results demonstrated that smokers are at higher risk to develop CAD, the odds ratio for CAD are almost two-fold higher for current smokers as compared to non-smokers, the odds ratio for CAD rise from 0.94 in those smoking less than 10 cigarettes per day to 5.02 in those smoking more than 10 cigarettes per day. Also, in multivariate logistic regression analysis, smoking remained as an independent predictor of coronary artery disease (OR 3.72; 95\% CI 2.08-6.70; $\mathrm{p}<0.01$ ) even after all other major risk factors had been taken into accounts. Pais et al. ${ }^{[10]}$, reported that Smoking $\geq 10$ cigarettes/day carries an independent four-fold increased risk of acute myocardial infarction. In India smoking is increasing particularly in the younger generation ${ }^{[11]}$. our study revealed that smoking ( $75 \%$ vs. $45 \%, \mathrm{p}<0.0001)$ were significantly higher in younger ( $\leq 45$ years) than in older $(>45$ years) patients as compared to controls. This suggested that smoking is one of the important risk 
factor for the causation of $\mathrm{CAD}$ in the younger population.

In developing countries $\mathrm{CAD}$ is thought to be predominantly a disease of the upper income groups. As development progresses this relation may reverse $^{[12]}$. Our findings show that cases of CAD had a lower income and lower education than controls accords with a cross-sectional study conducted in a rural area of Rajasthan in which the prevalence of ECG abnormalities were inversely related to the level of education ${ }^{[13]}$. Low education level may change the life style like diet rich in saturated fats which contribute to increase lipid and lipoprotein levels which may lead to coronary artery disease. Hardarson $^{[14]}$ and Kevin ${ }^{[15]}$ have stated that low education level can be considered as one of the independent risk factor for causing coronary heart disease. By contrast, Chadha and colleagues ${ }^{[16]}$, in a study from Delhi reported a lower prevalence of ECG abnormalities among people of a lower socioeconomic status than among those of a higher status. Our study suggests that Indian population is at equal risk for developing $\mathrm{CAD}$ like any other developed country.

There are only few studies on lipid levels among Indian patients with CAD living in India, and most of the reports are from South India ${ }^{[7,17]}$. In our study group, increased total cholesterol and LDLcholesterol were significantly associated with CAD, in accordance with similar observations made in earlier studies ${ }^{[18]}$. The TC and LDL-c levels in our patient group are lower (approximately 170-190 $\mathrm{mg} / \mathrm{dl}$ ) but still they develop angiographically proven CAD. These levels are far lower than those observed in migrant Indians, and certainly lower than in the patients of western ethnic origin ${ }^{[18]}$. These differences might be due to different dietary habits and lifestyles which suggest that Indians develop CAD at much lower TC and LDL-c levels, and may need appropriate revision in the ATP guidelines for lipid management if the nonlipid-lowering benefits of agents such as statins are to be utilized in Indian patients. HDL-cholesterol levels was found to be strongly and independently associated with CAD. The Framingham data suggested an optimal HDL-c level $>52 \mathrm{mg} / \mathrm{dl}$ in men and $>66 \mathrm{mg} / \mathrm{dl}$ in women ${ }^{[19]}$, which probably may not apply to our patients as they have much lower levels even in the absence of disease. It is possible that in the face of low HDLcholesterol, even modest elevation of LDL with consequent elevation of the LDL/ HDL and total cholesterol/ HDL-cholesterol ratio could contribute to atherogenesis in this population.

Univariate analysis showed that mean TG levels were significantly higher in patients with CAD than controls and in multivariate analysis TG is not found to be an independent risk factor of CAD, in accordance with similar observation made by Krishnaswami et $a l .{ }^{[20]}$. In our study, mean apolipoprotein B level was found to be strongly and independently associated with $\mathrm{CAD}$ and in multivariate analysis apo B was a stronger predictor of risk in CAD patients. In some studies from India, the percentage of patients below the age of 45 years suffering from acute myocardial infarction (AMI) is reported as high as $25-40 \%{ }^{[21]}$. Our study revealed that younger patients (age $\leq 45$ years), TC, LDL-C, and apolipoprotein B levels were significantly higher than in older patients (age $>45$ years). A study from South India showed that TC and TG levels had equal expression in the development of atherosclerotic disease, with cholesterol being of greater relevance in those $<50$ years and TG being of greater relevance in those $>50$ years $^{[20]}$.

Early CAD has previously been reported to be associated with family history ${ }^{[22]}$. Our results indicated that family history of CAD was significantly higher in younger ( $\leq 45$ years) patients, but the prevalence of hypertension and diabetes mellitus were significantly higher in older patients (>45years). Our results demonstrated diabetic patient with CAD had significantly higher levels of total cholesterol and triglycerides than non-diabetics patients with CAD. In the Framingham study, TG levels were higher and HDL-C levels were lower in diabetics when compared to non-diabetics ${ }^{[23]}$. Study by Ramachandran et al. ${ }^{[7]}$, had shown that the lipid profile of diabetic CAD patients had a higher concentration of TC, TG, LDL-c, LDL-c / HDL-c ratio, and a lower concentration of HDL-c, which is almost similar to our results. Test of proportion showed that the diabetic subgroup had a significantly higher prevalence of hypertension which could be one of the associated risk factor for CAD.

To conclude, our study suggests that, in addition to smoking and hypertension (which are important risk factors for CAD in most populations), diabetes and raised apolipoprotein $\mathrm{B}$ are important across a wide range of values in North Indians. Although, North Indians develop CAD at lower levels of cholesterol than other populations younger patients have a more atherogenic lipid profile than older patients. Hypertension is a strong risk factor in diabetic patients for the occurrence of CAD but a family history of premature CAD and smoking are the risk factors in younger patients. Our data suggest that stringent smoking cessation programmes, treatment of hypertension, the ATP-III guidelines for the treatment of dyslipidemia and improving the level of education are likely to have a profound effect on 
the burden of premature coronary artery disease in North India.

\section{ACKNOWLEDGEMENTS}

Supported by grant from the Uttar Pradesh Council for Science and Technology (UPCST), Lucknow, India.

\section{REFERENCES}

1. Dawn J., 1996. Coronary artery disease risks in Asian Indians. Curr. Opin. Lipidol., 7: 196-198.

2. Corral, J., R.C. Gonzalez, J.A. Iniesta, J. Rivera, C. Martinez and V. Vicente, 2000. The F XIII Val34Leu polymorphism in venous and arterial thromboembolism. Haematologica., 85: 293-7.

3. Gupta, R. and V.P. Gupta, 1996. Meta-analysis of coronary heart disease prevalence in India. Indian Heart J., 48: 241-245.

4. Chadha, S.L., S. Radhakrishnan, K. Ramachandran, U. Kaul and N. Gopinath, 1990. Epidemiological study of coronary heart disease in urban population in Delhi. Indian J. Med. Res., 92: 424-30.

5. Mckeigue, P.M., G.J. Miller and M.G. Marmot, 1989. Coronary heart disease in south Asians overseas: a review. J. Clin. Epidemiol., 42: $597-$ 609.

6. Mckeigue, P.M., J.E. Ferrie, T. Pierpoint and M.G. Marmot, 1993. Association of early onset coronary heart disease in South Asians men with glucose intolerance and hyperinsulinemia. Circulation., 87: 152-161.

7. Ramachandran, A., I. Sathyamurthy, C. Snehalatha, K. Satyavani, S. Sivasankari and J. Misra, 2001. Risk variables for coronary artery disease in Asian Indians. Am. J. Cardiol., 87: 267-271.

8. Reddy, K.S., 1994. Rising burden of cardiovascular disease in India. In: Sethi KK (Ed). Coronary artery disease in Indians. New Delhi: Cardiological Society of India., pp. 63-72.

9. Farmer, J.A. and A.M. Gotto, 1992. Risk factors for coronary artery disease. In: Braunwald E, ed. Heart disease. A textbook of cardiovascular medicine. $4^{\text {th }}$ ed. Philadelphia: WB Saunders., 1125-60.

10. Pais, P., P.M. Fay and S. Yusuf, 2001. Increased Risk of Acute Myocardial Infarction Associated with Beedi and Cigarette Smoking in Indians: Final Report on Tobacco Risks from a CaseControl Study. Indian Heart J., 53: 731-735.

11. Rissam, H.S., S. Kishore and N. Trehan, 2001. Coronary artery Disease in Young Indians- The Missing Link. J. Ind. Aad. Clin. Med., 2: 128132.
12. Davey, S.G., M.J. Shipley and G. Rose, 1990. Magnitude and cause of socioeconomic differentials in mortality: further evidence from the Whitehall study. J. Epidemiol. Commun. Health., 44: 265-70.

13. Pais, P., J.Pogue, H. Gerstein, E. Zachariah, D. Savitha, P.R. Nayak and S. Yusuf, 1996. Risk factors for acute myocardial infarction in Indians: a case-control study. Lancet., 348: 358363.

14. Hardarson, T., M. Gardarsdottir, K.T. Gudmundsson, G. Thorgeirsson, H. Sigvaldason and N. Sigfusson, 2001. The relationship between educational level and mortality. The Reykjavik study. J Intern Med., 249: 495-502.

15. Kevin, F. and F. Peter, 2004. Should Years of schooling be used to guide treatment of coronary risk factors? Annals FAM Med., 2: 469-473.

16. Chadda, S.L., S. Radhakrishnan, K. Ramachandran, U. Kaul and N. Gopinath, 1990. Epidemiological study of coronary hearth disease in a urban population of Delhi. Ind. J. Med. Res., 92: 424-430.

17. Mohan, V., R. Deepa, S. Shanthirani and G.J. Premalatha, 2001. Prevalence of coronary artery disease and its relationship to lipids in a selected population in South India: The CUPS study. J.Am.Coll. Cardiol., 38: 682-687.

18. Anand, S.S., S. Yusuf, V. Vuksan, S. Devanesen, K.K. Teo and P.A. Montague, 2000. Differences in risk factors atherosclerosis and cardiovascular disease between ethnic groups in Canada: the study of health assessment and risk in ethnic groups (SHARE). Lancet., 356: 279-284.

19. Abbott, R.D., P.W.F. Wilson, W.B. Kannel and W.P. Cestelli, 1998. High density lipoprotein cholesterol. Total cholesterol screening and myocardial infarction. The Framingham study. Arteriosclerosis., 8: 207-211.

20. Krishnaswami, S., G. Joseph and S.T. Chandy, 1996. Triglyceride expression. Int.J.Cardiol., 57: 167-171.

21. Girija, G., 1997. Risk factors profile of patients with acute MI. In Vijayaraghavar G (Ed). Cardiovascular Disease Prevention Trivandrum 78-83. American Heart Association Heart and stroke Statistical Update; 26-27.

22. Friedlander, Y., J.D. Kark and Y. Stein, 1985. Family history of MI as an independent risk factor for coronary heart disease. Br.Heart J., 53: 382-387.

23. Kannel, W.B., 1985. Lipids, diabetes and coronary heart disease: insight from Framingham study. Am Heart J., 110: 1100-1107. 